Original Article

Journal of Epilepsy Research pISSN 2233-6249 / eISSN 2233-6257

Received August 10, 2019

Revised September 21, 2019

Accepted January 31, 2020

Corresponding author:

Chun Kee Chung, MD, PhD

Department of Neurosurgery, Seoul

National University Hospital, 101

Daehak-ro, Jongno-gu, Seoul 03080, Korea

Tel. + 82-2-2072-2352

Fax. +82-2-744-8459

E-mail; chungc@snu.ac.kr

\title{
Clinical Relevance of Interictal Spikes in Tumor-Related Epilepsy: An Electrocorticographic Study
}

\author{
Changik Lee, MD', Woorim Jeong, $\mathrm{PhD}^{2,3}$, Chun Kee Chung, MD, PhD ${ }^{2,3,4}$ \\ ${ }^{1}$ Department of Neurosurgery, Seoul St. Mary’s Hospital, Seoul; ${ }^{2}$ Department of Neurosurgery, Seoul National \\ University Hospital, Seoul; ${ }^{3}$ Interdisciplinary Program in Neuroscience, Seoul National University College of \\ Natural Science, Seoul; ${ }^{4}$ Department of Brain and Cognitive Sciences, Seoul National University College of Natural \\ Sciences, Seoul, Korea
}

\begin{abstract}
Background and Purpose: Although some surgeons utilize interictal spikes recorded via electrocorticography (ECoG) when planning extensive peritumoral resection in patients with tumor-related epilepsy, the association between interictal spikes and epileptogenesis has not been fully described. We investigated whether the resection of interictal spikes recorded by ECoG is associated with more favorable surgical outcomes in tumor-related epilepsy.
\end{abstract}

Methods: Of 132 patients who underwent epilepsy surgery for tumor-related epilepsy from 2006 to 2013, seven patients who underwent extraoperative ECoG were included in this study. In each patient, ECoG interictal spike sources were localized using standardized low-resolution brain electromagnetic tomography and were co-registered into a reconstructed brain model. Correspondence to the resection volume was estimated by calculating the percentage of interictal spike sources in the resection volume. Results: All patients achieved gross total resection without oncological recurrence. Five patients achieved favorable surgical outcomes, whereas the surgical outcomes of two patients were unfavorable. Correspondence rates to the resection volume in the favorable and unfavorable surgical outcome groups were $44.6 \% \pm 27.8 \%$ and $43.5 \% \pm 22.8 \%$, respectively $(p=0.96)$. All patients had interictal spike source clusters outside the resection volume regardless of seizure outcome.

Conclusions: In these cases of tumor-related epilepsy, the extent of the resection of ECoG interictal spikes was not associated with postoperative seizure outcomes. Furthermore, the presence of interictal spike sources outside of the resection area was not related to seizure outcomes. Instead, concentrating more on the complete removal of the brain tumor appears to be a rational approach. (2019;9:126-133)

Key words: Electrocorticography, Surgery, Brain tumors, Interictal spike, Source localization

\section{Introduction}

Tumor-related epilepsy is pharmacoresistant in more than $50 \%$ of cases, and early surgical intervention is recommended. ${ }^{1}$ Previous studies reported that gross total tumoral resection is superior to subtotal resection. ${ }^{2}$ However, there is disagreement over the extent of surgery to enable seizure control while minimizing neurologic sequelae. Phi et al. ${ }^{3}$ showed tailored resection focusing on the tumor to be necessary for seizure control, even for tumors confined to the amygdala or parahippocampal gyrus. However, some investigators have advocated the extensive resection of the peritumoral cortex. ${ }^{4}$ Ghareeb and Duffau ${ }^{5}$ reported the significance of hippocampectomy in patients with paralimbic grade II glioma, even when the hippocampus had not been invaded.

Moreover, there has been some debate over the use of electrocorticography (ECOG) as an electrophysiological guide for tumor-related epilepsy surgery. Some surgeons utilize interictal epileptiform discharges recorded by intraoperative ECOG to delineate the resection margin. However, whether the use of intraoperative ECOG improves surgical outcomes is inconclusive. ${ }^{2}$ It has been suggested that the irritative zone, i.e., the region generating frequent interictal spikes, identified via subdural electrodes, needs to be resected along with the tumor considering that the infiltrated peritumoral neocortex may function as a key structure in epileptogenesis. ${ }^{4}$ In contrast, an- 
other group reported equivalent rates of seizure control irrespective of the use of intraoperative $\mathrm{ECOG}$, undermining the use of intraoperative ECOG in tumor-related epilepsy surgery. ${ }^{6,7}$

Several previous studies have investigated the usefulness of irritative zone removal in tumor-related epilepsy., ${ }^{2,7,8}$ However, those studies simply compared surgical outcomes of gross total tumor removal alone with those of extended surgery involving the removal of the irritative zone, and the spatial relationship between the interictal spikes and the resection volume has not been investigated, which may help to determine usefulness of interictal spikes recorded by ECOG in delineation of resection margin in tumor-related epilepsy. Regarding this is sue, the extraoperative ECoG, rather than intraoperative ECoG, provide a unique opportunity to comprehensive comparison of spatial relationship between interictal spikes of ECoG and resection volume, since it could provide 3-dimensional interictal spike source locations in the brain model rather than the position of the electrodes on the 2-dimensional cortical surface. Since all study patients who conducted extraoperative ECoG underwent computerized tomography (CT) after subdural electrode insertion, accurate determination of the electrode locations on the 3-dimensional space was possible. Moreover, epileptiform activity can be altered under anesthetic condition ${ }^{9}$ and extraoperative ECOG allows the longer observation periods in broader areas, which could help to achieve a comprehensive understanding of the relationship between irritative zone and epileptogenic zone in tumor-related epilepsy.

Therefore, in order to investigate whether the inclusion of interictal spikes recorded by ECOG to the resection margin could improve surgical outcomes in tumor-related epilepsy, we used interictal spike data from extraoperative ECoG and applied source localization technique. Correspondence between interictal spike sources and the resection volume was then compared according to the surgical outcome of tumor-related epilepsy patients.

\section{Methods}

\section{Patient profile}

Based on a final diagnosis of focal epilepsy with a single brain tumor, we selected consecutive patients who underwent extraoperative ECoG and epilepsy-related surgery from 2006 to 2013. All patients were operated on by the senior author. Patients' clinical data were retrospectively reviewed. The present study was approved by the Institutional Review Board of Seoul National University Hospital.

One hundred thirty-two patients were finally diagnosed, based on a pathologic examination, as tumor-related epilepsy during the study period. Although it is not routinely used for a presurgical evaluation, eight of 132 patients underwent extraoperative ECOG for one or more of the following reasons: 1) since the magnetic resonance imaging (MRI) findings were ambiguous to interpret as typical brain tumor, non-tumor related epilepsy could not be excluded; 2) disagreement over the suspected epileptogenic zone among epileptologists, thus requiring further investigation; and 3) a lesion close to the eloquent cortex. One of these patients was excluded because the number of inserted electrodes was deemed too small for a proper analysis (three $1 \times 4$ grids on temporal lobe). Thus, seven patients were included in the present study. Their surgical outcomes were evaluated by applying Engel's classification. ${ }^{10}$ An Engel I was consid-

Table 1. Patient profiles

\begin{tabular}{|c|c|c|c|c|c|}
\hline Pt. & $\begin{array}{c}\text { Sex/age } \\
\text { (years) }\end{array}$ & $\begin{array}{l}\text { Duration of } \\
\text { illness (years) }\end{array}$ & $\begin{array}{c}\text { PostOp F/U } \\
\text { (months) }\end{array}$ & $\begin{array}{c}\text { Tumor } \\
\text { involvement }\end{array}$ & Reason for invasive monitoring \\
\hline G1 & $\mathrm{M} / 25$ & 3 & 115 & Lt. ant-lat $\mathrm{T}$ & The MRI finding was not corresponded with typical brain tumor \\
\hline G2 & $M / 24$ & 14 & 98 & Lt. ant-lat $\mathrm{T}$ & The MRI finding was not corresponded with typical brain tumor \\
\hline G3 & $M / 25$ & 3 & 78 & Rt. post-lat $\mathrm{T}$ & The MRI finding was not corresponded with typical brain tumor \\
\hline G4 & $M / 27$ & 20 & 58 & Rt. O & $\begin{array}{l}\text { 1. The MRI finding was not corresponded with typical brain tumor } \\
\text { 2. Very broad epileptogenic zones (Rt. T-P-O) were suspected when using } \\
\text { noninvasive modalities }\end{array}$ \\
\hline G5 & $\mathrm{F} / 42$ & 22 & 32 & Lt. med T & $\begin{array}{l}\text { 1. Hippocampal sclerosis was the likely suspect on MRI } \\
\text { 2. Interictal spikes were widely dispersed from ant to post T on EEG and MEG }\end{array}$ \\
\hline B1 & $\mathrm{M} / 51$ & 15 & 117 & Rt. post-lat $\mathrm{T}$ & The MRI finding was not corresponded with typical brain tumor \\
\hline B2 & $\mathrm{F} / 46$ & 20 & 25 & Lt. post-lat T & $\begin{array}{l}\text { 1. The lesion on MRI was close to the eloquent cortex } \\
\text { 2. Discrepant results were obtained from noninvasive modalities }\end{array}$ \\
\hline
\end{tabular}

Pt., patient; PostOp, post-operative; F/U, follow-up; M, male; Lt., left; ant, anterior; lat, lateral; $T$, temporal lobe; MRI, magnetic resonance imaging; Rt., right; O, occipital lobe; P, parietal lobe; F, female; med, medial; EEG, electroencephalography; MEG, magnetoencephalography. 
ered a favorable outcome, whereas Engel II-IV were considered unfavorable outcomes.

The patient group consisted of five men and two women with ages ranging from 24 to 51 years (mean \pm standard deviation, 34.3 211.6 ) at the time of surgery. Patients' illness duration was between 3 and 22 years (mean, 13.9 \pm 7.9). All patients had taken at least three anti-epileptic drugs before being considered for epilepsy surgery. Brain tumors were located in the lateral temporal lobes in five patients and in the medial temporal and occipital lobes in one patient each. The patient with the occipital lobe brain tumor (G4) had undergone previous brain tumor resection; the second surgery was due to seizure recurrence and a residual tumor (Table 1).

\section{Invasive monitoring and epilepsy surgery}

Subdural electrodes were placed primarily on the lesion detected via MRI and surrounding areas, including the eloquent cortex. Additional electrodes were placed based on the results from other noninvasive evaluation modalities. The inter-electrode distance was $10 \mathrm{~mm}$, and the electrode diameter was $4 \mathrm{~mm}$. After electrode implantation, electrode positions were recorded by means of CT. All patients' ECoG tracings were continuously recorded using a multichannel digital electroencephalography (EEG) acquisition system (Telefactor; Grass Technologies, West Warwick, RI, USA) for at least 24 hours until sufficient seizure events were observed.

Determination of the extent of surgery was based on the location of the tumor and the ictal onset zone as determined with extraoperative $\mathrm{ECOG}^{3}$ The ictal onset zone included those electrode positions that first showed sustained rhythmic ECOG changes that could be distinguished from background or interictal activities. ${ }^{11}$ When the tumor involved the hippocampus, an anterior temporal lobectomy with

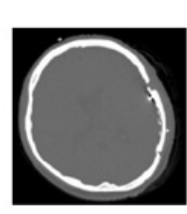

Brain CT after electrode insertion

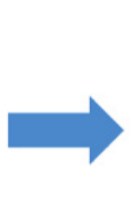

ion

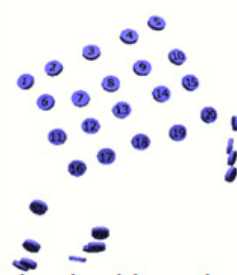

Subdural grids and strips

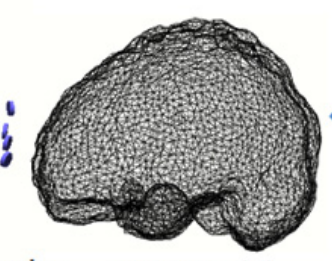

BEM model

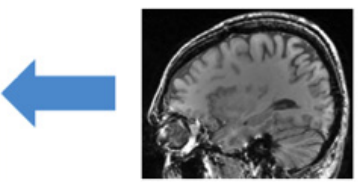

Preoperative MR

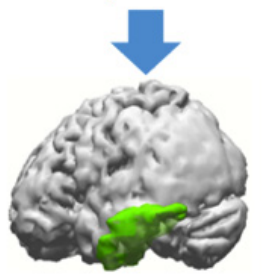

Segmented cortex with the resection volume delineated by postoperative MR

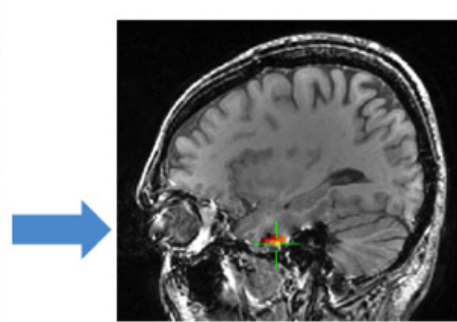

Source localization using sLORETA

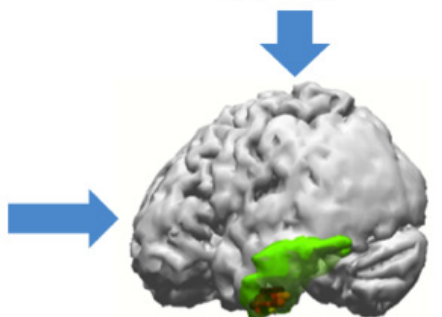

Coregistration of source locations into the segmented cortex

Figure 1. A schematic illustration of the procedures of ECoG source localization of interictal spikes using the case of the patient G1. All visually detected ECoG interictal spikes (indicated by either red or black arrows) were used for source localization. The result of interictal spike source localization indicated by the red arrow is described. Following the source localization, the coordinates of each calculated source were registered into the individual's segmented cortex to establish the interictal spike source distribution. The resection volume in the segmented cortex, marked with green, was delineated with postoperative MRI. CT, computerized tomography; BEM, boundary element model; MR, magnetic resonance; ECoG, electrocorticography; sLORETA, standardized low-resolution brain electromagnetic tomography; MRI, magnetic resonance imaging. 
amygdalohippocampectomy along with resection of the ictal onset zone was conducted. In other cases, the resection volume was delineated based on the lesion identified via MRI and the ictal onset zone. ${ }^{3}$ Following surgery, the patients underwent follow-up visits in an outpatient clinic for at least 25 months (mean, $74.7 \pm 37.7$ ).

\section{ECoG source localization of interictal spikes}

The procedure for the ECoG source localization of interictal spikes is illustrated in Fig. 1. Preoperative T1-weighted sagittal MRI was used for segmentation of the cortex and reconstruction of a single-compartment boundary element model (BEM), a realistic volume conductor of an individual brain. The positions of the subdural grids and strips were manually detected using each patient's axial CT slices, and their coordinates were projected onto the relevant volume conductor. A 10 minutes interictal period showing frequent spikes with small artifacts was selected from the entire extraoperative ECOG monitoring period, and the peak values of all visually detected interictal spikes in the selected period were used for source localization. This approach was used because the highest spatial resolution can be obtained from the peak values. ${ }^{12}$ Signals recorded by depth electrodes were not used for source localization because the potentials generated by depth electrodes are not suitable for a BEM model. ${ }^{13}$ Three $1 \times 4$ depth electrodes were inserted into the right temporal lobe of patient $\mathrm{G} 3$, but the data acquired from the depth electrodes was not utilized during the delineation of the resection margin. For these processes, standardized low-resolution brain electromagnetic tomography (SLORETA) with the Curry 7.0 software (Compumedics NeuroScan, Charlotte, NC, USA) was used. ${ }^{14}$ Because sLORETA returns a current distribution rather than a single dipole, the point with the maximum F-distribution value was selected for assessment, as it is assumed to be the position best fitting the actual source location. ${ }^{15}$
Following the source localization of all interictal spikes within the selected period, the coordinates of each calculated source were registered into the individual's segmented cortex to establish the interictal spike source distribution. Correspondence to the resection volume was estimated by determining the percentage of interictal spike sources located within the resection volume as delineated by postoperative T1-weighted MRI. We also examined whether patients had interictal spike sources exclusively clustered in the resection volume, or had other source clusters outside the resection volume.

\section{Results}

Of the seven patients in this study, five patients (71.4\%) achieved a favorable outcome (G1-G5) and two patients had an unfavorable outcome (B1, B2). The patients' profiles and the reasons for the extraoperative ECOG are shown in Table 1. Gross total tumor resection was achieved in all patients, and no tumor recurrence was observed during the follow-up visits. Patients G3 and G5, diagnosed as having a World Health Organization grade III malignant tumor, did not receive additional therapy. Instead, they were annually evaluated by means of brain MRI for tumor recurrence. Based on a presurgical evaluation, patient G5 was diagnosed with hippocampal sclerosis, but after surgical resection this patient was finally diagnosed with dual pathology (anaplastic astrocytoma and hippocampal sclerosis).

All patients' ictal onset zones were included in the resection volume except G3 and G4. For patient G3, the ictal onset zone was detected adjacent to the brain tumor, but the surgery was not extended to the ictal onset zone. Instead, the operator concentrated on complete tumor resection. The ictal onset zone of patient G4 was widely dispersed from the posterior temporal lobe to the occipital lobe. The resection margin in this case was limited to the brain tumor because

Table 2. Postoperative outcome

\begin{tabular}{lcccl}
\hline Pt. & Types of surgery & Engel & COR $(\%)$ & \\
\hline G1 & L & I & 63.6 & Mixed tumor (ganglioglioma, gangliocytoma, DNET), FCD type IIB \\
G2 & L & I & 71.2 & Glioneuronal tumor \\
G3 & L & I & 0 & Anaplastic ganglioglioma \\
G4 & L & I & 47.4 & Ganglioglioma \\
G5 & ATL+AH & I & 40.7 & Anaplastic astrocytoma with HS, FCD type IIIA, IIIB \\
B1 & L & III & 27.3 & Ganglioglioma \\
B2 & L & IV & 59.6 & Pleomorphic xanthoastrocytoma \\
\hline
\end{tabular}

Pt., patient; COR, correspondence to the resection volume; L, lesionectomy; DNET, dysembryoplastic neuroepithelial tumor; FCD, focal cortical dysplasia; ATL, anterior temporal lobectomy; $\mathrm{AH}$, amigdalohippocampectomy; HS, hippocampal sclerosis. 
total resection of the entire ictal onset zone may have resulted in severe neurologic sequelae.

Types of surgery, outcomes, rates of correspondence of interictal spike sources to the resection volume, and the pathologic results of each patient are shown in Table 2. The source localization results of patients with favorable and unfavorable outcomes are shown in Figs.
2 and 3, respectively. Ictal onset zones are also delineated in the figure. All patients had interictal spike source clusters outside of the resection volume. The rates of correspondence to the resection volume among the patients with favorable and unfavorable outcomes were $44.6 \% \pm 27.8 \%$ and $43.5 \% \pm 22.8 \%$, respectively $(p=0.96)$.
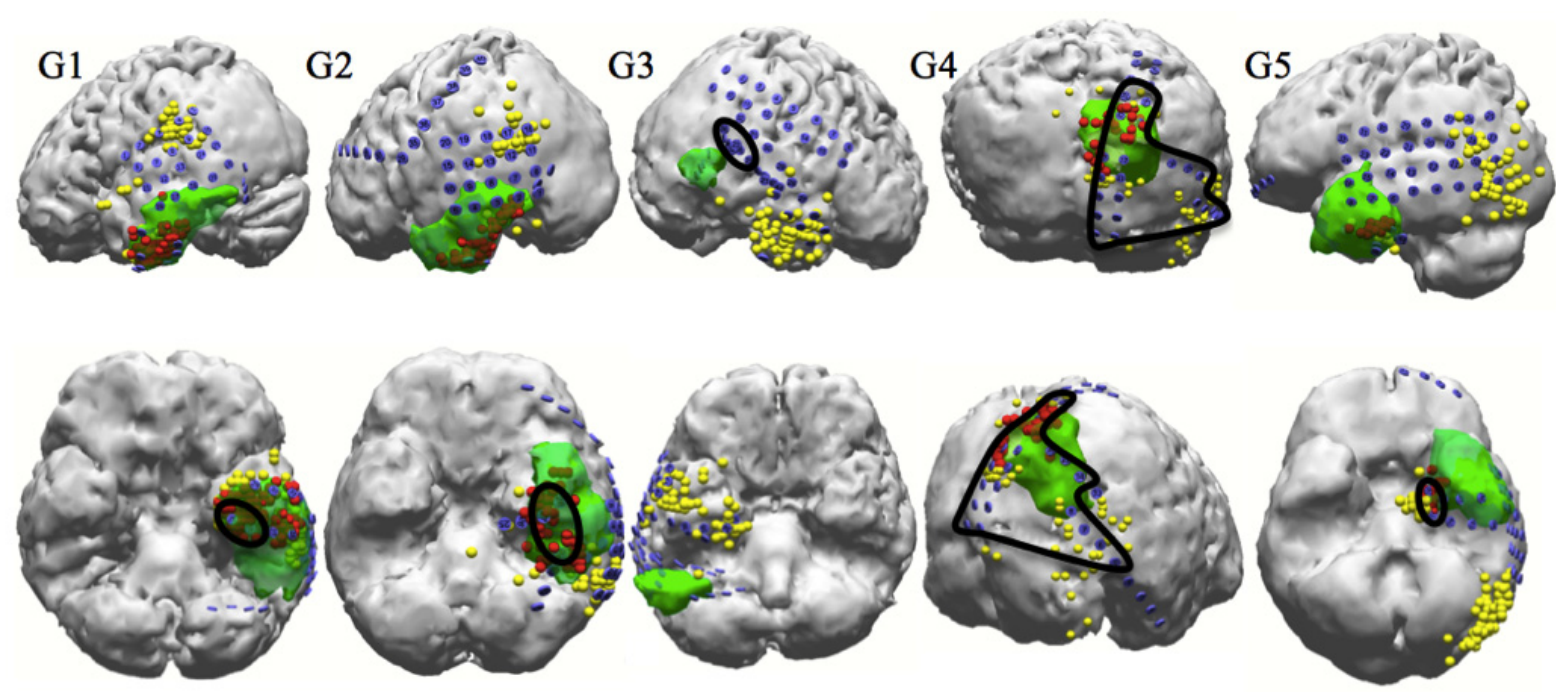

Figure 2. Interictal spike source localization results from tumor-related epilepsy patients with favorable surgical outcome. The figures on the second row indicate the same patients of the first row shown from the different direction. The ictal onset zone is delineated with black line. The resection volume is marked with green. The numbered blue dots indicate electrodes. Each red sphere represents an interictal spike source located inside the resection volume, and each yellow sphere represents an interictal spike source outside of the resection volume.
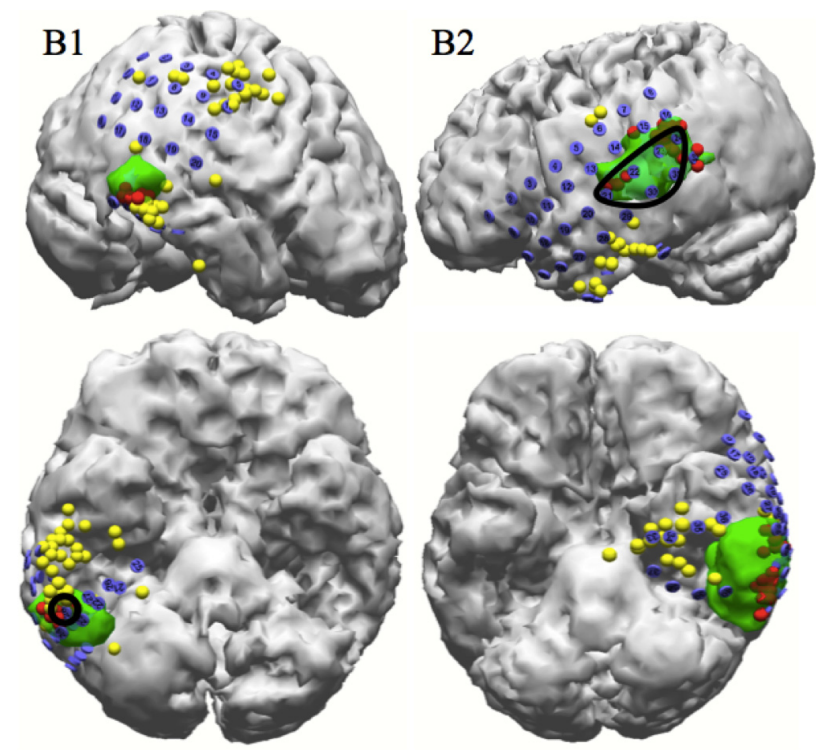

Figure 3. Interictal spike source localization results from tumor-related epilepsy patients with unfavorable surgical outcome. The figures on the second row indicate the same patients of the first row shown from the bottom. 


\section{Discussion}

In the present study, we analyzed ECoG-identified interictal spike sources in seven tumor-related epilepsy patients. Interictal spike source clusters were found both inside and outside the resection volume irrespective of the surgical outcome, and the differences between the rates of correspondence relative to the resection volume were not significant between the favorable and unfavorable surgical outcome groups. These results extend the findings of previous scalp EEG-based studies which showed that the irritative zone in tumor-related epilepsy tends to be extensive and can even be located on the contralateral side. ${ }^{16}$

Interictal spike patterns of patients with tumor-related epilepsy appear to differ from those of non-tumor-related epilepsy patients. Our previous study had analyzed 32 temporal lobe epilepsy patients who underwent both extraoperative ECOG monitoring and epilepsy surgery in Seoul National University Hospital from 2006 to $2010 .^{17}$ In that study, patients with favorable surgical outcomes tended to have concentrated interictal spike source clusters located exclusively in the resection volume, whereas concentrated source clusters tended to be located outside the resection volume in patients with unfavorable surgical outcomes, thus suggesting that interictal spike sources are closely associated with the epileptogenesis and may help delineate the resection volume.

However, of the seven patients in the present study with interictal spike source clusters remote from their brain tumors, leaving spike clusters unresected did not result in unfavorable surgical outcomes in five patients. Based on this result, we speculate that spurious interictal activities occurring near the tumor or in peritumoral tissue operate via a mechanism different from that associated with seizure onset. Therefore, resection of areas with spurious interictal spikes is not necessary. Some interictal spike sources appear to reflect epileptic activities, as they overlap with the resection volume. However, discrimination of interictal spikes relevant to ictogenesis from those unrelated to ictogenesis may not be simple, making the use of interictal spikes during the delineation of the resection volume implausible.

Such a difference may indicate the presence of different mechanisms linking interictal spikes and seizures. There may be genes uniquely associated with seizure onset and others uniquely associated with interictal spikes. ${ }^{18}$ If this is the case, these two phenomena may require distinct therapeutic approaches. A previous study reported that rats generating interictal spikes were induced by injecting tetanus toxin into somatosensory cortex. They showed layer-2/3-spe- cific CREB activation; ${ }^{19}$ this has also been observed in an interictal state of both tumor- and non-tumor-related epilepsy patients. ${ }^{20,21}$ In the rat study, administration of a MAPK inhibitor prevented layer-2/3 CREB activation and significantly reduced the frequency of interictal spikes, but there were no improvements in seizure scores. ${ }^{19}$ Although this study did not consider tumor-related epilepsy, it is suggested that interictal and ictal activities utilize different molecular pathways.

Tumor-related mass effects that induce increased intracranial pressure, focal ischemic damage, acidosis, and disruption of the blood-brain barrier may also explain the different results obtained from non-tumor-related epilepsy patients. ${ }^{1}$ Focal cortical dysplasia (FCD), the second most common cause of refractory chronic partial epilepsy, ${ }^{22}$ is frequently associated with brain tumors. Our group previously reported that $32 \%$ of patients with ganglioglioma had peritumoral architectural abnormalities associated with FCD. ${ }^{23}$ FCD adjacent to a tumor may influence epileptogenesis. It was reported that tumors accompanied with FCD tended to have higher seizure frequencies than those associated with solitary tumors. ${ }^{24}$ Additional resection of the cortex when it exhibits FCD has been asserted as necessary for seizure control, ${ }^{25}$ although this was contradicted by another study. ${ }^{26}$ In the present study, given that the pathologic findings of only two patients (G1 and G5) showed FCD adjacent to a tumor, the influence of FCD on epileptogenesis could not be assessed. In addition, because cortical dysplasia is usually found near a tumor, the presence of interictal spikes distant from the tumor could not be explained by the presence of peritumoral cortical dysplasia.

Of the five patients with favorable outcomes, two patients' ictal onset zones were not completely resected (G3 and G4, in Fig. 2). It is possible that the ictal onset zone may not have been precisely delineated by subdural electrodes, as subdural electrodes cannot detect activity which originates at the subcortical level. ${ }^{27}$ Because the ictal onset zone of patient $\mathrm{G} 4$ was observed broadly to include the occipital lobe to the posterior temporal lobe, subdural electrodes may have detected propagated activities rather than the actual ictal onset. However, complete tumor resection led to seizure freedom despite leaving a wide ictal onset zone unresected. Therefore, we suggest that complete tumor resection is more important than the resection of the ictal onset zone recorded from extraoperative ECoG in tumor-related epilepsy.

The small number of patients analyzed in the present study inevitably limits our capacity to fully describe the features of tumor-related epilepsy. Although the identification of the ictal onset zone via extraoperative ECoG following subdural electrode placement has 
been considered as the gold standard in determining the resection area of non-tumor-related epilepsy, extraoperative ECoG has rarely been used in tumor-related epilepsy, as the primary goal of this type of surgery is not making seizure free but maximal tumor resection while minimizing neurologic sequelae. ${ }^{6,17,27}$ In addition, the presence of a subdural electrode in situ for several days increases the risk of increased intracranial pressure; the most formidable complication associated with intracranial mass lesions. In our institute, the patients did not undergo extraoperative ECoG when the brain tumor was highly suggested by MRI. In case of the seven patients in the present study, the equivocal MRI findings could not exclude non-tumor-related epilepsy, and tumor-related epilepsy was finally diagnosed after pathologic examination. Therefore, performing extraoperative ECoG in tumor-related epilepsy patients was unavoidably rare.

Notwithstanding, due to its advantages of extraopertive ECOG over intraoperative ECoG, which provides more accurate electrode locations, longer monitoring period, and broader area on the cortex, we could compare the relationship between removal of interictal spikes from ECoG and the surgical outcome in tumor-related epilepsy patients. Further study with more subjects and longer follow-up periods is necessary to identify the spatial and functional relationship between interictal spikes and brain tumors.

In the present study, ECoG interictal spike sources were localized in seven tumor-related epilepsy patients. In all patients, tumor resection margins were delineated by MRI results and the ictal onset zone determined by extraoperative ECoG. The difference between the rates of correspondence of the interictal spike sources to the resection volume in the favorable and unfavorable surgical outcome groups was not significant. All patients had interictal spike source clusters outside of the resection volume. While some interictal spike sources overlapped the resection volume, the post-surgical presence of nonresected interictal spike sources was not associated with an unfavorable surgical outcome. We conclude that some interictal spike sources tend to reflect spurious activities associated with the brain tumor; thus, the inclusion of interictal spike sources in the resection volume may be unwarranted. Complete tumor resection is more important than the use of ECoG.

\section{Acknowledgements}

The manuscript has been presented in oral presentation session, 23rd Korean Epilepsy Congress on June 18th, 2018 at Yongsan City Hotel, Seoul, Korea.

This research was supported by the Basic Science Research
Program through the National Research Foundation of Korea, funded by the Ministry of Science \& ICT (2016M3C7A1904984).

\section{Conflicts of Interest}

The authors declare that they have no conflict of interest.

\section{References}

1. Rudà R, Bello L, Duffau H, Soffietti R. Seizures in low-grade gliomas: natural history, pathogenesis, and outcome after treatments. Neuro Oncol 2012;14 Suppl 4:iv55-64.

2. Chang EF, Potts MB, Keles GE, et al. Seizure characteristics and control following resection in 332 patients with low-grade gliomas. J Neurosurg 2008; 108:227-35.

3. Phi JH, Kim SK, Cho BK, et al. Long-term surgical outcomes of temporal lobe epilepsy associated with low-grade brain tumors. Cancer 2009; 115:5771-9.

4. Pallud J, Capelle L, Huberfeld G. Tumoral epileptogenicity: how does it happen? Epilepsia 2013;54(Suppl 9):30-4.

5. Ghareeb F, Duffau H. Intractable epilepsy in paralimbic Word Health Organization Grade II gliomas: should the hippocampus be resected when not invaded by the tumor? I Neurosurg 2012;116:1226-34.

6. Englot DJ, Berger MS, Barbaro NM, Chang EF. Predictors of seizure freedom after resection of supratentorial low-grade gliomas. A review. J Neurosurg 2011;115:240-4.

7. Englot DJ, Han SJ, Berger MS, Barbaro NM, Chang EF. Extent of surgical resection predicts seizure freedom in low-grade temporal lobe brain tumors. Neurosurgery 2012;70:921-8; discussion 928.

8. Qiu B, Ou S, Song T, et al. Intraoperative electrocorticography-guided microsurgical management for patients with onset of supratentorial neoplasms manifesting as epilepsy: a review of 65 cases. Epileptic Disorder 2014;16:175-84.

9. Mittal S, Barkmeier D, Hua J, et al. Intracranial EEG analysis in tumor-related epilepsy: evidence of distant epileptic abnormalities. Clin Neurophysiol 2016;127:238-44.

10. Engel JJ, Van Ness P, Rasmussen T, Ojemann LM. Outcome with respect to epileptic seizures. In: Engel JJ, ed. Surgical treatment of the epilepsies. 2nd ed. New York: Raven Press, 1993;609-22.

11. Lee SK, Lee SY, Kim KK, Hong KS, Lee DS, Chung CK. Surgical outcome and prognostic factors of cryptogenic neocortical epilepsy. Ann Neurol 2005;58:525-32.

12. Zhang $Y$, van Drongelen $W$, Kohrman $M$, He B. Three-dimensional brain current source reconstruction from intra-cranial ECOG recordings. Neuroimage 2008;42:683-95.

13. Dumpelmann $M$, Fell J, Wellmer J, Urbach $H$, Elger CE. 3D source localization derived from subdural strip and grid electrodes: a simulation study. Clin Neurophysiol 2009;120:1061-9.

14. Pascual-Marqui RD. Standardized low-resolution brain electromagnetic 
tomography (sLORETA): technical details. Methods Find Exp Clin Pharmacol 2002;24 Suppl D:5-12.

15. Wagner $M$, Fuchs $M$, Kastner J. Evaluation of SLORETA in the presence of noise and multiple sources. Brain Topogr 2004;16:277-80.

16. Hamer HM, Najm I, Mohamed A, Wyllie E. Interictal epileptiform discharges in temporal lobe epilepsy due to hippocampal sclerosis versus medial temporal lobe tumors. Epilepsia 1999;40:1261-8.

17. Lee C, Kim JS, Jeong W, Chung CK. Usefulness of interictal spike source localization in temporal lobe epilepsy: electrocorticographic study. Epilepsy Res 2014;108:448-58.

18. Mittal S, Shah AK, Barkmeier DT, Loeb JA. Systems biology of human epilepsy applied to patients with brain tumors. Epilepsia 2013;54 Suppl 9:35-9.

19. Barkmeier DT, Senador D, Leclercq K, et al. Electrical, molecular and behavioral effects of interictal spiking in the rat. Neurobiol Dis 2012; 47:92-101.

20. Rakhade SN, Yao B, Ahmed S, et al. A common pattern of persistent gene activation in human neocortical epileptic foci. Ann Neuro/ 2005;
58:736-47

21. Phi JH, Kim SK. Clinical pearls and advances in molecular researches of epilepsy-associated tumors. J Korean Neurosurg Soc 2019;62:313-20.

22. Chung CK, Lee SK, Kim KJ. Surgical outcome of epilepsy caused by cortical dysplasia. Epilepsia 2005;46 Suppl 1:25-9.

23. Im SH, Chung CK, Cho BK, et al. Intracranial ganglioglioma: preoperative characteristics and oncologic outcome after surgery. I Neurooncol 2002; 59:173-83.

24. Cossu M, Fuschillo $D$, Bramerio $M$, et al. Epilepsy surgery of focal cortical dysplasia-associated tumors. Epilepsia 2013;54 Suppl 9:115-22.

25. Chassoux F, Rodrigo S, Mellerio C, et al. Dysembryoplastic neuroepithelial tumors: an MRI-based scheme for epilepsy surgery. Neurology 2012;79: 1699-707.

26. Nolan MA, Sakuta R, Chuang N, et al. Dysembryoplastic neuroepithelial tumors in childhood: long-term outcome and prognostic features. Neurology 2004;62:2270-6.

27. Duffau $\mathrm{H}$. Brain mapping in tumors: intraoperative or extraoperative? Epilepsia 2013;54(Suppl 9):79-83. 